\title{
Editorial: Data Assimilation of Nonlocal Observations in Complex Systems
}

\begin{abstract}
A. Hutt ${ }^{1 *}$, M. Bocquet ${ }^{2}$, A. Carrassi ${ }^{3,4}$, L. Lei ${ }^{5}$ and R. Potthast ${ }^{6}$
${ }^{1}$ Team MIMESIS, INRIA Nancy Grand Est, Strasbourg, France, ${ }^{2}$ CEREA, Joint Laboratory École Des Ponts ParisTech and EdF $R \& D$, Université Paris-Est, Champs-sur-Marne, France, ${ }^{3}$ Dept of Meteorology and National Centre for Earth Observations, University of Reading, Reading, United Kingdom, ${ }^{4}$ Mathematical Institute, Utrecht University, Utrecht, Netherlands, ${ }^{5}$ The School of Atmospheric Sciences, Nanjing University, Nanjing, China, ${ }^{6}$ Department of Mathematics and Statistics, University of Reading, Reading, United Kingdom
\end{abstract}

Keywords: covariance localization, ensemble Kalman filter, Kalman smoother/filtering, satellite data, meteorology

\section{Editorial on the Research Topic}

\section{Data Assimilation of Nonlocal Observations in Complex Systems}

Natural complex systems exhibit spatio-temporal dynamics on multiple scales that are difficult to predict and understand. Prominent examples for such systems are the Earth atmosphere, the brain or even single biological cell. To gain deeper insights into the system's dynamics and to be able to predict their evolution, observations are used to motivate, inform, or possibly infer models of the system. Today more and more observations of diverse types are available and mirror the dynamics of systems. In general, one may distinguish two types of data. The so-called in-situ or local observations represent measurements obtained on the system itself, such as temperature in the atmosphere at a specific location or electric potentials in biological cells. Other observations are not point-wise nor associated to a spatial location but represent integrals of the system's activity. Examples in meteorology for such nonlocal observations are satellite radiances, slant delays or radar reflectivities. In biological systems, non-invasive measurement techniques provide nonlocal observations, such as electro- and magneto-encephalogram or Magnetic Resonance Imaging. Together with observations, realistic models are essential to improve the understanding of natural complex systems and to predict their dynamical evolution.

To merge both models and observations, it is essential to develop techniques that estimate the activity of the system with well-adapted methods. Data assimilation is an important technique to optimally match experimental data with an underlying model. Typical goals of data assimilation are the optimal estimation of model parameters or providing accurate initial conditions to achieve skillful forecasts of the system's dynamics. In recent years numerous and increasingly diverse observation techniques have been developed and implemented to capture integrals of the system's activity and, hence, data assimilation of nonlocal observations has become increasingly important.

The Research Topic has brought together recent theoretical works in data assimilation of nonlocal observations with an emphasis on specific applications. Evensen et al. have investigated in detail a recently developed iterative ensemble smoother and proposed a simple derivation of the algorithm together with practical applications in (oil) reservoir history matching. This paper serves as a useful guide for researchers who intend to implement an iterative ensemble data assimilation algorithm for operational, i.e., professional, purpose.

Ensemble Kalman filters (EnKFs) are powerful methods to merge high-dimensional nonlinear models and nonlocal observations. Due to the large model dimension (may be in the order of $10^{5}$ and beyond), typical setups utilize a number of ensemble members (typically 100) that is much lower 
than the model dimension, or even of the unstable subspace of the dynamics. To balance this sub-sampling, one typically reduces the model space by using localization, be domain localization or covariance localization. If local observations are easily assimilated with local EnKFs, with good performance, nonlocal observations impose additional complexity since they cannot be distributed among local domains. Various localization techniques have already been developed and applied successfully. Covariance localization can handle nonlocal observations at the cost of a much more computationally demanding EnKF update. To alleviate this numerical cost, Farchi and Bocquet have considered augmented ensembles to implement covariance localization. The authors have discussed some major difficulties related to the implementation of augmented ensembles. In particular, they have proposed and evaluated the use of random singular value decomposition to efficiently sample the Schur product of the sample forecast covariance matrix. Bishop et al. have complemented this work with a detailed discussion which methodological elements resemble previously published elements.

These latter studies represent important contributions to improve data assimilation of nonlocal observations, while these have not been addressed explicitly. This is done in the work by van Leeuwen that addresses explicitly the impact of nonlocal observations and how they influence data assimilation results. The author argues that, in general, the current research focus on covariance modeling should shift from the prior covariance to covariances between model and observations. It is recommended that future research should optimize the covariance structures between observations and model variables. The work of Hutt et al. considers a similar approach by empirical application of covariance localization to satellite data in a regional model. Here, the vertical covariance localization radius is equal to the width of the satellite sensitivity function and thus locally adapted at each data assimilation update. The authors have empirically found that this choice yields the lowest first guess prediction errors. Similarly, Wang et al. apply the global group filter to experimental tropical cyclone data. This adaptive filter estimates an optimal localization width and vertical location for each channel of every satellite platform with varying meteorological conditions.
Most of the methods considered in this Research Topic belong to the family of the (ensemble) Kalman filter (EnKF) that assumes Gaussian-shape prior and posterior probability distributions of the system state. This EnKF framework permits to describe probability densities by mean and covariance only which, however, yield a bias and limits the data assimilation performance. This is true especially in the cases of nonlinear models, nonlinear observation operators or nonlocal observations. To this end, variational approaches, iterative filter, or particle filter methods may outperform local EnKFs if the probability density functions do not resemble a Gaussian distribution. Brankart has proposed a specific sampling method based on a Monte Carlo Markov Chain to design a global, possibly non-Gaussian, probability density. The author points out that this method is supposed to suit better to nonlocal observations and nonlocal dynamics than other methods. Here the key element is that the posterior distribution may be nonGaussian without any assumptions on its shape.

\section{AUTHOR CONTRIBUTIONS}

$\mathrm{AH}$ has conceived the structure and all authors have written the manuscript.

\section{ACKNOWLEDGMENTS}

CEREA is a member of Institut Pierre-Simon Laplace.

Conflict of Interest: The authors declare that the research was conducted in the absence of any commercial or financial relationships that could be construed as a potential conflict of interest.

Copyright (c) 2021 Hutt, Bocquet, Carrassi, Lei and Potthast. This is an open-access article distributed under the terms of the Creative Commons Attribution License (CC BY). The use, distribution or reproduction in other forums is permitted, provided the original author(s) and the copyright owner(s) are credited and that the original publication in this journal is cited, in accordance with accepted academic practice. No use, distribution or reproduction is permitted which does not comply with these terms. 